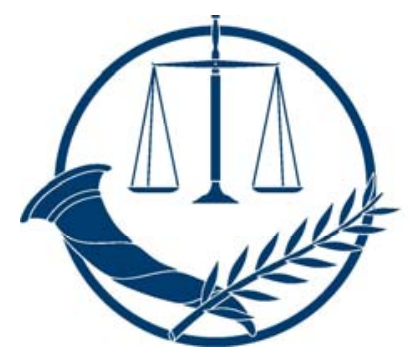

IAAEU

Institut für Arbeitsrecht und Arbeitsbeziehungen in der Europäischen Union

IAAEU Discussion Paper Series in Economics

No. $02 / 2013$

\title{
Variants of the Monoamine Oxidase A Gene (MAOA) Predict Free-riding Behavior in Women in a Strategic Public Goods Experiment
}

Vanessa Mertins, Andrea B. Schote, Jobst Meyer

\section{February 2013}

Institute for Labour Law and Industrial Relations in the European Union (IAAEU) 


\title{
Variants of the monoamine oxidase A gene (MAOA) predict free-riding behavior in women in a strategic public goods experiment
}

\author{
Vanessa Mertins ${ }^{1} \&$ Andrea B. Schote ${ }^{2} \&$ Jobst Meyer ${ }^{3}$
}

\begin{abstract}
Laboratory experiments have documented substantial heterogeneity in social preferences, but little is known about the origins of such behavior. Previous research on public goods experiments suggests that individual-level demographic and psychological variables correlate with player types. However, the key question about biological sources of variation in these preferences remains open. The aim of this study is to uncover genetic variations that influence differences in cooperative behavior. For this reason, we identify types of players within a strategic public goods experiment. We explicitly test for an association between individual variance in strategy choice and the functional promoter-region repeat of the monoamine oxidase $A$ gene (MAOA). Our experimental findings suggest a link between $M A O A$ and the occurrence of free-riding in females. Females with $M A O A-L$ are less likely to behave like weak free-riders than $M A O A-H$ carriers, whereas among males, our results did not support a significant relation between genotype and player type. Furthermore, $M A O A-L$ female carriers contribute more than $M A O A-H$ subjects to the public good if they know that others contribute nothing, and they showed slightly lower scores on the Machiavellianism scale. This is the first piece of evidence that genotype might predict player type within a public goods setting. It contributes to our understanding of biological drivers of economic decision-making and points to the need for further exploration.
\end{abstract}

Keywords gene; player type; public good; conditional cooperation; experimental economics

JEL Classification H41; D87; C91; C72

PsycINFO classification 2500; 2200; 3000

\footnotetext{
${ }^{1}$ Institute for Labour Law and Industrial Relations in the European Union (IAAEU), University of Trier, Trier, Germany, email: mertins@iaaeu.de (corresponding author).

${ }^{2}$ Department of Neurobehavioral Genetics, Institute of Psychobiology, University of Trier, Trier, Germany, email: schotefrese@uni-trier.de.

${ }^{3}$ Department of Neurobehavioral Genetics, Institute of Psychobiology, University of Trier, Trier, Germany, email: meyerjo@uni-trier.de.
} 


\section{Introduction}

A puzzle of great interest to biologists, economists or psychologists is the often observed large-scale cooperation with genetic strangers. Indeed, robust experimental findings in the lab and in the field point to a strong willingness of the average individual to contribute sizeable shares of an endowment, although free-riding on others' contributions is the rational, payoff-maximizing strategy. Furthermore, recent studies suggest that most people follow the social norm of "conditional cooperation" (Chaudhuri, 2011; Fehr \& Fischbacher, 2004; Fischbacher \& Gächter, 2010): contributions to public goods depend directly on how other group members behave. At the same time, individuals with multiple behavioral types exist, each of them involving a distinctive behavioral pattern (Brosig, 2002; Burlando \& Guala, 2005; Fischbacher et al., 2001; Kurzban \& Houser, 2005; Ostrom, 2000). What we still do not know is whether there are biological sources of variation in these preferences for cooperation. This study considers the possibility that genotype predicts player type (e.g., conditional cooperators, free-riders) within a strategic public goods experiment. By means of an incentivized controlled laboratory experiment, we rigorously test the impact of a particular gene on individuals' preferences towards cooperation.

Many factors, including gender, culture, and age, have been considered explanatory variables for cooperative behavior. Gender alone fails to predict cooperation. By referring to a multitude of studies on behavior in public goods games, Croson and Gneezy (2009) concluded that there are inconsistent results regarding gender-dependent cooperation, with women being more or less cooperative than men in various studies. Cross-cultural studies comparing the distribution of player types and individual preferences across different countries confirm that cultural background influences one's attitude towards cooperation (see e.g., Anderson et al., 2011; Burlando \& Hey, 1997). Herrmann and Thöni (2009), for example, showed that the cultural background, rather than the socio-economic environment within a society, has an effect on people's preferences towards cooperation. Kocher et al. (2008) found that conditional cooperation was prevalent in subject pools from three different continents. The distribution of player types (e.g., conditional cooperators, free-riders) as well as the extent of conditional cooperation, however, differs across countries. Andersen et al. (2008) provided evidence that a society's structure is critically linked to its provision of public goods. They find that matrilineal societies not only have fewer strong free-riders, but also the highest level of public goods provision.

These results provide support for the argument that human groups largely differ in their social norms, but the key question as to whether these norms are learned or have a more 
fundamental basis remains open. By conducting experiments with young children, Fehr et al. (2008) gained insight into the developmental origins of social preferences. Their results showed that at the ages of 3-4, the majority of children behave selfishly, while with increasing age, children follow the social norm of inequality aversion.

Although all of these findings are important in understanding and modeling behavior in social dilemma situations, they do not explain the observed differences. In search of the ultimate forces behind individual differences in preferences towards cooperation, economists started to take the idea seriously that "nature" matters as well. A steadily increasing number of studies have examined the link between economic preferences and hormones (e.g., Apicella et al., 2011; Buser, in press), or genetic markers (e.g., Beauchamp et al., 2011). Hormones such as testosterone and cortisol mediate behavior by either increasing or decreasing the probability that an individual will express a certain behavior by acting on the neuronal mechanisms underlying the behavior (Soares et al., 2010). Recently, women treated with testosterone were found to experience a disruption in cooperative behavior together with an increase in self-oriented and more egocentric behavior (Wright et al., 2012). Besides hormones' rapid effects on the functional level due to changes in the neural circuit, hormones and neurotransmitters can have long-lasting effects by changing an individual's phenotype during prenatal and early childhood developmental stages (Soares et al., 2010). Those behavioral traits are likely to determine one's social behavior and can also be influenced by genetic and epigenetic differences (Liu et al., 1997; Meaney, 2001). Empirical findings from a field called "genoeconomics" (Benjamin et al., 2007; Beauchamp et al., 2011; Navarro, 2009) suggest that this holds true for economic behavior as well. One strand of research focuses on twin studies, investigating the impact of genetic factors on economically relevant behavior in monozygotic (identical) and dizygotic (fraternal) twin pairs raised in identical environments. Researchers have identified a significant degree of genetic influence on variables such as income (Taubman 1976), education (Behrman \& Taubman, 1989), political attitudes (Alford et al., 2005), cooperativeness in trust games (Cesarini et al., 2008), bargaining behavior (Wallace et al., 2007), the tendency to be self-employed (Nicolaou \& Shane, 2010), risk preferences (Zhong et al., 2009b), and preferences for giving and taking risks (Cesarini et al., 2009a). These studies have found that economic preferences have a genetic component, with heritability ${ }^{4}$ estimates ranging from 18 to $42 \%{ }^{5}$ However, in public goods games the

\footnotetext{
${ }^{4}$ Genetics uses the concept of "heritability" to describe the proportion of the variability on a trait that is contributed by genes. Cesarini et al. (2008), for example, reported a heritability estimate of $20 \%$ in a trust game with Swedish twins. Heritability of $20 \%$ does not mean that trusting behavior is $20 \%$ determined by genes.
} 
underlying biological sources for variation and the impact of genetics on economic behavior are still largely unknown.

Another strand of literature builds upon recent improvements in individual gene identification, which have allowed us to study particular genetic factors that influence a given trait, i.e., a single quantifiable measurement of an organism, such as an individual's attitude towards economic risk taking (Zhong et al., 2009a). Genetic association studies attempt to identify the occurrence - more often than expected by chance — of a trait together with a given genetic variant (i.e., a given version of a gene, known as an allele) in a population. Targeted studies focus on "candidate genes," which have been identified based on previously reported associations.

When studying the association between genetic variants and economic traits, researchers are increasingly relying upon experimental games. Several recent targeted studies have followed this approach and have provided evidence of an association between economic preferences and particular genes. Knafo et al. (2008) showed that fund allocation in the dictator game was, in part, determined by length of the arginine vasopressin la gene (AVPR1a) RS3 promoter-region repeat. Kuhnen and Chiao (2009) found that variants of two genes (5-HTTLPR and DRD4 repeat) that regulate dopamine and serotonin neurotransmission and which were previously linked to emotional behavior, anxiety and addiction, were significant determinants of risk-taking behavior in investment decisions. Dreber et al. (2009) also found this association between DRD4 and risk preferences, but Dreber et al. (2011) reported this association only among men and not women, and Frydman et al. (2010) failed to replicate this association. De Neve et al. (2012) found a correlation between 5-HTTLPR and subjective well-being in their initial study and mixed results in their replication study. Israel et al. (2009) demonstrated that genetic polymorphisms for the oxytocin receptor (OXTR) were associated with pro-social fund allocation in the dictator game. However, Apicella et al. (2010) could not replicate these findings albeit their larger sample size. Zhong et al. (2009a) found that carriers of the low variant of the monoamine oxidase A gene (MAOA-L) are more likely to exhibit risky behavior. This finding has been substantiated by Frydman et al. (2011), who showed that these individuals only engage in more risky behavior when it is advantageous to do so. The authors concluded that their results provide a cautionary tale on

Rather, it means that if all subjects were raised in the exactly the same environment, we would see $20 \%$ of the variability we see now.

${ }^{5}$ Navarro (2009) noted that heritability measures of socio-economic variables are of similar magnitude to those of physical occurrence of common diseases. 
the interpretation of previous behavioral results related to the monoamine oxidase A gene (MAOA).

Three important insights follow from these prior findings and have inspired the present study. First, targeted studies generally appear to be quite valuable and promising in identifying particular genes that might be associated with economic decision-making. Indeed, as much of the behavioral neuroscience literature points to the serotonergic, dopaminergic, and noradrenergic systems' important role in decision-making, it seems justified to focus initially on one of the genes involved in the transport, binding, or degradation of these neurotransmitters. Second, we need to be aware that the replication of candidate gene studies might be difficult due to the polygenetic architecture of those traits and the small effects of individual genes on that given trait (discussed in Benjamin et al., 2012). Third, subjects with similar preferences might still make quite different choices, depending on the behavioral specificity of their defined phenotype (Frydman, 2011). Thus, using simple but accurate preference elicitation procedures, which eliminate difficulties resulting from complex decision environments such as repeated play or strategic interaction, may allow for an improved interpretation of previous results. In this study, we shed further light on the previously reported association between voluntary contributions towards a public good and MAOA (Mertins et al. 2011) by allowing the same subjects to make additional choices using a different procedure. These findings may help to get a clearer picture of the link between cooperation preferences and this particular gene.

MAOA encodes the enzyme monoamine oxidase A that degrades neurotransmitters such as serotonin, dopamine and epinephrine (Hariri et al., 2005). Different variants of it regulate transcription, metabolism, and signal transfer between neurons, all of which have an effect on social interactions (Craig, 2007; Spitzer et al., 2007). In particular, a variable number of tandem repeats (VNTR) in the promoter-region of MAOA leads to high and low activity variants. Alleles with 3.5 and four copies are transcribed more efficiently than those with three or five copies. The less transcriptionally efficient alleles are called MAOA-L, and the more efficient are called MAOA-H (Sabol et al., 1998). MAOA-L has been found to be associated with various kinds of antisocial behavior, including violence and aggression, both in the field (Caspi et al., 2002) and in the lab (Gallardo-Pujol et al., forthcoming). McDermott et al. (2009), for example, show an association between MAOA-L in males and behavioral aggression following provocation (i.e., high take rates by proposers in the power-to-take game). Aggression and cooperation may represent two sides of the same coin in child development and are two critical features in children's social domain. In combination with 
maltreatment in children, lower MAOA expression predicts aggression (Kim-Cohen et al., 2006), whereas it might be associated with normal behavior in children that were in good hands. On the other hand, a less active promoter might result in higher neurotransmitter levels in the brain, which might lead to more cooperative behavior. In a murine knockout model (Cases et al., 1995), mice completely lacking MAOA were found to be highly aggressive. However, mice with normal MAOA levels (independent on the VNTR) displayed the whole range of species-specific behavior with respect to social relationships, and they displayed intense social investigation, which are usually highly cooperative among clan members. Additionally, brain imaging studies have shown an activation of similar brain areas in aggression (Nelson \& Trainor, 2007) and cooperation (Decety et al., 2004) suggesting similar underlying neuronal mechanisms that are determined by genetic and environmental factors. Furthermore, brain functions during cognition, emotional arousal, personality test-takings, and working memory activation (Meyer-Lindenberg et al., 2006; Rommelse et al., 2008; Ziermann et al., 2012) as well as the function and anatomy of specific brain regions in the limbic system (reviewed in Nordquist \& Oreland, 2010) were affected by the MAOA VNTR.

Recently, we showed a genetic association between MAOA and individuals' behavior in a repeated public goods game (Mertins et al., 2011). As we aimed to study decision-making in conditions of uncertainty, the setting was a dynamic one that offered subjects increasing information about group members' cooperativeness over the course of the game. We found that MAOA-L male subjects held more pessimistic beliefs about others' cooperativeness than MAOA-H male carriers, resulting in fewer contributions to the provision of the public good. With decreasing uncertainty about others' behavior, however, the genetic effect diminished. For females, an opposite pattern was observed: there was no genetic influence in the early rounds, but a strong genetic influence in late rounds of the game. Furthermore, MAOA-L female carriers held more optimistic beliefs about others' willingness to cooperate, and accordingly, contributed more than $M A O A-H$ females. In line with these findings, recently, the MAOA-L was related to higher levels of happiness in women but not in men suggesting that MAOA-L women may be more sensitive to positive aspects of the environment and less susceptible to stressors (Chen et al., 2012).

Our present study using the same subjects as in the previous paper differs from the former one in terms of the decision procedure. By using the strategy method (Selten, 1967), participants stated contingent responses for each information set, i.e., they were asked to indicate their complete strategy: each player decided how much to contribute to the public good for all feasible group contributions. Therefore, we focused on players' strategic behavior 
and excluded any confounding factors resulting from variations in expectations. To this end, we replicated Fischbacher et al.'s (2001) one-shot experiment with anonymous interaction partners, which enabled us to measure subjects' preferences unaffected by potential confounds. There is an ongoing debate by experimentalists as to whether the strategy method and direct elicitation procedures produce different results. The use of the strategy method may force participants to think about each set of information in a different way than if they could primarily concentrate on each set as it arises throughout the course of the game (Roth, 1995). For instance, highly emotional responses may be suppressed: it is plausible that people will have stronger emotional reactions to actual observed actions than to hypothetical actions that might be taken ("hot" versus "cold" environment). Laboratory findings have been mixed. Brandts and Charness (2000) found no behavioral difference between hot and cold settings, whereas Brosig et al. (2003) and Güth et al. (2001) do. Thus, it seems warranted to explore the relationship between cooperative player types and MAOA in a cold environment to either corroborate our prior research in the hot setting or to identify differences between both environmental settings.

Our study provides new insights in several respects. This is the first study on the association of a particular candidate gene and cooperativeness in a strategic public goods experiment. Linking the assessed genotype with the observed player type may allow us to derive evidence of a potential genetic basis for different behavioral patterns. Whereas most of the recent $M A O A$ association studies have focused on phenotypes with negative connotations such as antisocial or aggressive behavior, our design permits the study of a complete range of feasible actions (including positive behaviors) that are directly dependent on others' behavior. Little research so far has considered the association between molecular genetics and voluntary cooperation. Furthermore, the vast majority of association studies have used self-reported questionnaires only. We are among the first to combine methods and techniques of experimental economics and behavioral genetics and to use data from questionnaires to confirm our findings. Last, our subject pool consists of both genders, whereas most MAOA studies have used males only (see e.g., Garllado-Pujol, forthcoming; McDermott et al., 2009). However, it is necessary to consider males and females separately, as recent findings have suggested that the pattern of associations between genotypes of MAOA in females differ from males. ${ }^{6}$ In particular, our previous findings (Mertins et al., 2011) suggested significant gender

\footnotetext{
${ }^{6}$ For example, female subjects carrying the MAOA- $H$ showed a higher risk of being high alcohol consumers, whereas among men, MAOA- $L$ was related to higher alcohol consumption (Nilsson et al., 2010). Among girls with psychosocial risk, $M A O A-H$ confers an increased risk for criminal behavior, whereas among men, the low activity variant and social risk interacts to predict criminal activity (Sjöberg et al., 2007).
} 
differences in the association between MAOA and cooperativeness. Those differences might be due to hormones such as testosterone and estrogen and their receptors, which act on gene regulation and subsequent brain development in a gender-specific manner (Hines, 2011; Kuo et al., 2010). Additionally, MAOA has an androgen response element in its promoter region, which can be occupied by testosterone bound androgen receptors (Ou et al., 2006). A significant interaction effect of high testosterone levels and MAOA on antisocial behavior has been shown only in male MAOA-L carriers (Sjoberg et al., 2008). Therefore, higher testosterone levels in men could compensate the MAOA- $L$ effect seen in women leading to an increased likelihood of negative MAOA-L effects in men. In women, those effects will not be compensated due to the much lower levels of testosterone. Third, estrogens play an important role as a neuromodulative reagent and influences the dopaminergic neurons in the brain (Riecher-Rössler \& de Geyter, 2007), which may have an additional positive effect in MAOA$L$ females.

In the present study, we found some experimental support for the notion that MAOA is weakly associated with cooperation and the occurrence of free-riding in females: Females with $M A O A-H$ are less cooperative than $M A O A-L$ carriers and show a significantly higher risk of being weak free-riders. Furthermore, there are several suggestive patterns in the questionnaire data, which merit further exploration in the link between $M A O A-L$ and cooperativeness in females. In males, we did not find evidence to suggest an association between genotype and player type. This is the first study to link player types derived from contributions to the public goods in an incentivized strategic game to a specific gene. It complements recent findings on the impact of genetics on economic preferences and provides starting points for further investigations of biological modulators of economic behavior.

\section{Experimental Design and Procedures}

To elicit subjects' preferences within a public goods game, we replicated Fischbacher et al.'s (2001) experiment ${ }^{7}$. In this game, the strategy method (Selten, 1967) was used in which subjects indicated, in an incentive-compatible way, their willingness to contribute to the public good, depending on the other group members' average contributions. In this one-shot linear public goods experiment, subjects were endowed with 20 tokens and were randomly divided into groups of four. Each subject decided how much of the endowment to keep and

\footnotetext{
${ }^{7}$ As we used exactly the same protocol, we refer the reader for any details of the design to the original source and to the appendix. The design has recently been used, e.g., by Fischbacher and Gächter (2010) and Herrmann and Thöni (2009). Subjects played the strategic game (reported here), and the repeated game afterwards (reported in Mertins et al., 2011). Since the game under consideration was played first, no behaviorally relevant interferences need to be accounted for.
} 
how much to contribute to a "project," the public good. The payoff function was given by the following equation:

$$
\pi_{i}=20-g_{i}+0.4 \sum_{j=1}^{4} g_{j}
$$

with $g_{i} \in\{0,1, \ldots 20\}$ being individual $i$ 's contribution to the public good. The sum of all group members' contributions was multiplied by the factor 1.6 and the outcome equally shared among all group members, independent of individual contributions. As the private marginal return of any token contributed to the public good was 0.4 , rational selfish players were expected to contribute nothing.

Subjects were first asked to fill out a contribution table, that is, to decide on their own contribution to the public good dependent on the other group members' average contributions (21 choices, as the other group members' average contributions were rounded to integers). This setting allows individual levels of cooperativeness to be measured in the presence of other group members who, on average, completely free ride, contribute a small, medium, or large share of their endowment, or even contribute their full endowment. The contribution table yields complete strategy profiles for each subject and thus allows players to be classified according to type. In addition, subjects chose one unconditional contribution and indicated what they thought the others would contribute. At the end of the experiment, a random device chose one group member who was paid according to the contribution table. The remaining three subjects were paid following their unconditional contribution and therewith defining the payoff for the randomly chosen group member.

The comparative advantage of this experimental design is that we measured individuals' complete preferences over the whole strategy space in the absence of uncertainty regarding others' behavior. That is, we obtained data on any contingent state of the world: for each individual, we observe his/her reactions to other group members' average decisions, but expectations about others' behavior did not play a role in subjects' decisions. Besides, averages might mask considerable heterogeneity among individuals. An approach which addresses this concern is given by player type classification. Following Fischbacher et al. (2001), we considered four distinct player types: free-riders (who contributed nothing), conditional cooperators (who raised contributions if the average contributions of the other members increased); hump-shaped or triangle cooperators (who had a hump-shaped contribution pattern as a function of others' average contributions) and erratic (who had very 
irregular contribution patterns). These types have been proven to occur in robust shares (Fischbacher \& Gächter, 2010) and are stable over time ${ }^{8}$ (Fischbacher et al., 2001).

As a second measure of cooperative player types, we elicited the individual levels of Machiavellianism (Christi \& Geis, 1970) using the German version (see Shajek, 2007) of a 20-item questionnaire. Machiavellianism has been considered a combination of selfishness and opportunism (Spitzer et al., 2007). Subjects indicated their degree of agreement with statements such as "Never tell anyone the real reason you did something unless it is useful to do so," and, "Most people are basically good and kind." Following Kurzban and Houser (2001) and Gunnthorsdottir et al. (2002), people with low levels of Machiavellianism (low Machs) are more likely to conform to social norms to pursue their self-interest. High Machs, on the other hand, are individuals who tend to be opportunistic and rational, and thus are expected to free-ride. In short, low Machs are expected to be more cooperative than high Machs.

As said before, we had to consider male and female subjects separately because significant gender $\mathrm{x}$ genotype interaction effects have been observed in previous studies. Based upon our previous results using the same subject pool (Mertins et al. 2011), we might expect that female carriers of $M A O A-L$ would be more cooperative than $M A O A-H$ subjects (as observed in late rounds in our repeated public goods game). Accordingly, MAOA-L female subjects should be less susceptible to play the free-riding strategy in the actual game. For male subjects, our previous results suggest no association between MAOA and player type. Although we had found a highly significant association between MAOA-H and contributions in early rounds of the repeated public goods experiment, no link was observable in late rounds when uncertainty about others' behavior diminished. An environment with low uncertainty most closely resembles our strategic setting, thus we should not expect to observe an association between MAOA and cooperativeness for male subjects. To test whether after all such a link either for males or females exists, we applied two-sided tests throughout the paper.

As previously reported (Reif et al., 2008), both men and women were assigned to two groups: male carriers of 4 repeats were assigned to $M A O A-H$, carriers of 3 repeats to $M A O A-L$ (see Appendix for MAOA genotyping). The MAOA is located on the $\mathrm{X}$ chromosome; therefore in men the 3/4 genotype does not exist. The high activity group of female subjects consists of $3 / 4$ and $4 / 4$ genotypes, the low activity group of 3/3 genotype. Within our subject pool of 33 males and 63 females, this assignment resulted in $33 \%$ of MAOA-L men (which is equivalent

\footnotetext{
${ }^{8}$ To test for stability, Fischbacher et al. (2001) asked their subjects in a post-experimental questionnaire again to fill out a hypothetical "contribution table." The results were almost identical to the schedules submitted in the actual experiment; therefore type classification has test-retest reliability.
} 
to the average proportion found in Western subject pools) and $19 \%$ of MAOA- $L$ women (five subjects were excluded from the analysis due to genotyping problems). All participants were non-related, randomly chosen Caucasians derived mainly from the ethnically homogenous Frankonian population of Rhineland-Palatia, Germany. All alleles tested were found to be in the Hardy-Weinberg equilibrium. Thus, we can exclude population stratification as a putative bias.

As control variables, we gained self-reported data on a wide range of individual characteristics $^{9}$ such as subjects' risk attitudes, patience, and impulsivity on an 11-point scale, with zero indicating the lowest possible levels of their willingness to take risks, patience, and impulsivity. We used the same wording of the questions as in the 2004 wave of the German Socio-Economic Panel Study. Also, subjects completed a 15-item (Germanlanguage) version of the well-established NEO-FFI developed by Costa and McCrae (1989) (see Gerlitz \& Schupp, 2005 on the implementation and reliability of this short version of the personality test), a personality questionnaire indicating individuals' five main personality dimensions (the so-called "Big Five": conscientiousness, neuroticism, openness to experience, agreeableness, and extroversion). In addition, we collected survey measures on individuals' reciprocal inclinations from the 2005 wave of the German Socio-Economic Panel (see Dohmen et al., 2009 for details). Furthermore, we used the German version of a questionnaire (Graf, 2004) to measure self-monitoring (Snyder 1974). In addition, we gathered additional socio-demographic data including participants' age, gender, and major in university.

The experiment was conducted at Trier University. Subjects were recruited with ORSEE (Greiner, 2004). Ninety-six subjects took part in the experiment, which consisted of four sessions with 24 subjects each. All participants were students from various fields. Instructions were read aloud, and then subjects had to solve several control questions before the experiment started. The experiment was computerized using z-tree (Fischbacher, 2007). Individuals were separated from the rest of the participants by sitting in cubicles. As this experiment was the first in a sequence of experiments, there were no interference effects from previous subjects' decision-making. Subjects first completed the experimental tasks and the post-experimental questionnaire. Thereafter, people were genotyped using buccal swabs and Listerine mouth wash. Participants gave informed written consent prior to participating. The study was approved by the university's ethics committee. The experiment (including genotyping) lasted about 40 minutes and subjects earned an average of $€ 8.91$ (including a show-up fee of $€ 5)$.

\footnotetext{
${ }^{9}$ The questionnaires are available from the corresponding author upon request.
} 


\section{Results}

\subsection{Overview}

Figure 1 shows the average contributions, according to the contribution table, for female (Fig. 1A) and male subjects (Fig. 1B) who were classified as MAOA-L or MAOA-H carriers. For females, MAOA-L carriers nearly fit the 45-degree line of perfect conditional cooperation up to an average group contribution of $50 \%$ (i.e., 10 tokens), whereas $M A O A-H$ carriers contributed less. On a descriptive level, we see that MAOA-L female carriers contributed more, especially in a highly uncooperative environment. However, there were no statistically significant differences $(p \geq 0.059)$ between $M A O A-L$ and $M A O A-H$ for any group contribution level using a two-sided Mann-Whitney test (M-W test). Classifying others' contributions into three rough categories (low contributions: 0-6, medium contributions: 714, high contributions: 15-20) also yielded no significant differences between MAOA-L and $M A O A-H(p \geq 0.056)$. The same was true for the contribution levels of MAOA-H (mean = $7.085)$ and $M A O A-L($ mean $=8.130)$ females averaged over all feasible contributions of others $(\mathrm{p}=0.321, \mathrm{M}-\mathrm{W}$ test $)$.
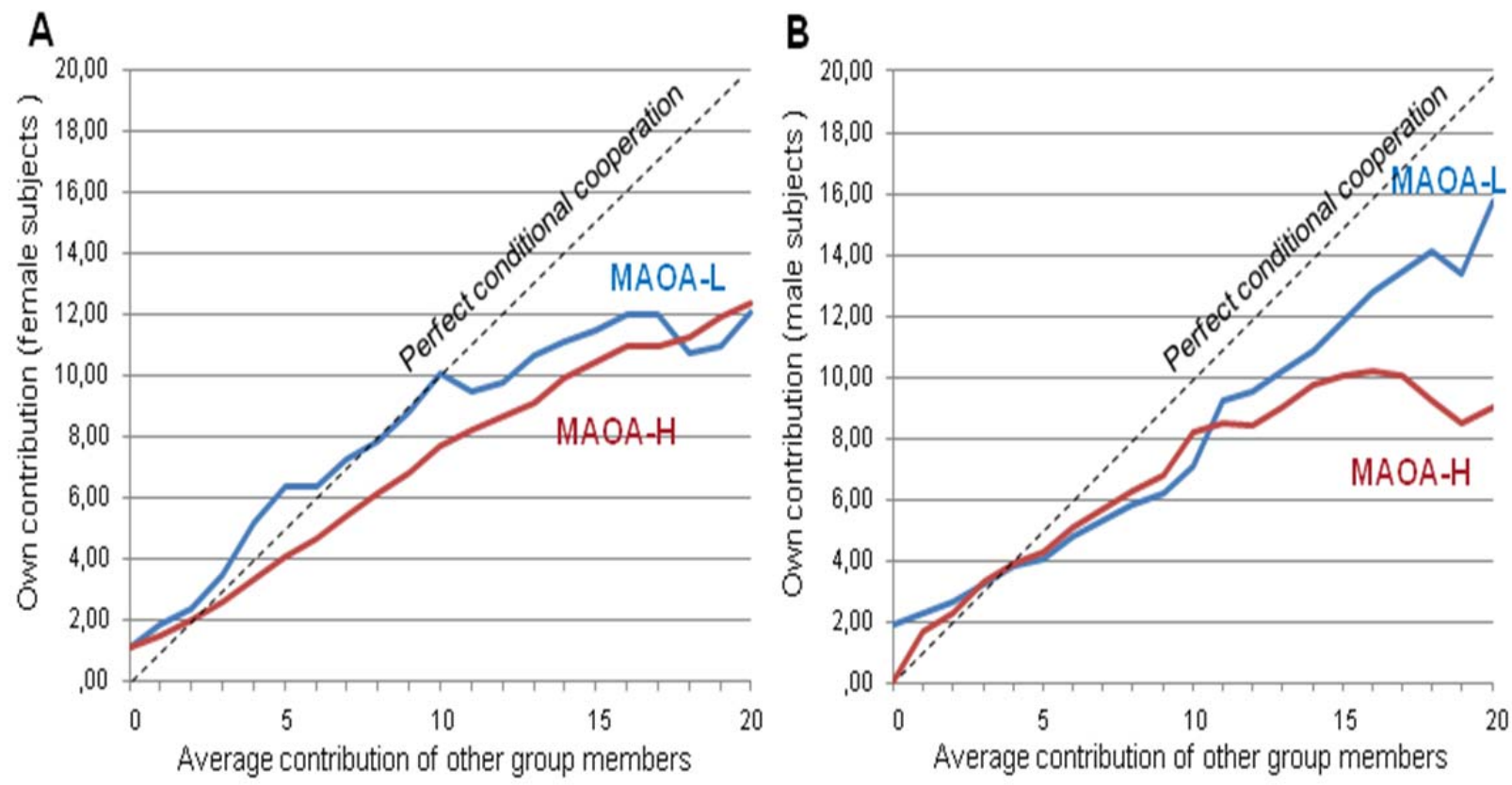

Figure 1. Subjects' average contributions dependent on other group members' average contributions

For males, Figure 1B shows that the average contributions of MAOA-L and MAOA-H subjects are strikingly identical up to an average cooperation level of others of about $75 \%$. Beyond that, MAOA-L and MAOA-H carriers developed in opposite directions: whereas $M A O A-L$ subjects still followed an increasing path (parallel to the ideal line of perfect 
conditional cooperation), $M A O A-H$ subjects held their contributions constant for several given contributions of others and ultimately decreased them. For high group contributions, MAOA-L male carriers seemed to be more cooperative than $M A O A-H$ carriers, but there was no significant difference according to the Mann-Whitney test. For example, in cases where subjects were facing a maximum mean contribution of 20 from other group members, MAOA$H$ subjects contributed 9.05 tokens on average, whereas MAOA- $L$ carriers contributed 15.73 tokens. ${ }^{10}$ This difference was, however, not significant ( $p=0.057$, two-sided). The same was true for the contribution levels of MAOA-H (mean = 6.693) and MAOA-L $($ mean $=8.022)$ males averaged over all feasible contributions of others $(p=0.192)$.

A feasible approach, considering the heterogeneity in contribution preferences, is to compare the slopes and intercepts of linear regressions. To this end, we computed them for all subjects. For male subjects, we did not find a significant difference between the slopes for MAOA-L $($ mean $=0.705)$ and $M A O A-H($ mean $=0.460, p=0.205)$ subjects. The same was true for the female subjects carrying MAOA-L $($ mean $=0.543)$ and $M A O A-H($ mean $=0.591, p=0.820)$. Similarly, no significant difference was detected in intercepts for males (MAOA-L mean $=$ 0.971; MAOA-H mean $=2.097, p=0.203$ ). For female subjects, however, genotype made a slight difference. Whereas the average individual intercept of linear regression was as high as 2.700 for $M A O A-L$ genotypes, the mean was 1.178 for $M A O A-H$ females. The difference was significant with $p=0.04$, pointing to stronger cooperativeness of $M A O A-L$ genotypes in cases where others did not contribute.

\subsection{Distribution of Player Types}

Next, we turn to the analysis of the complete individual contribution schedules. Table 1 shows the distribution of male player types according to Fischbacher et al.'s (2001) classification, Table 2 the respective data for females. First, we tested whether MAOA impacts the frequency of player types. We found that the distribution of types did not differ significantly between MAOA- $L$ and MAOA-H, neither for the male $(p=0.135)$ nor the female subjects $(p=0.213)$ using the conservative Fisher's exact test.

We now turn to a detailed analysis of male player types. As can be seen in Table 1, the share of free-riders among MAOA-H (4.5 percent) and MAOA-L (18.2 percent) did not differ significantly across genetic predispositions when using the Fisher's exact test $(p=0.252)$. Similarly, unconditional contributions and beliefs did not differ $(p>0.221, \mathrm{M}-\mathrm{W}$ test).

\footnotetext{
${ }^{10}$ Note that differences in the corner of a distribution should generally not be over-interpreted, as players might attach only a small probability to the notion that these states of nature are decisive.
} 
Table 1: Distribution of Male Player Types Separated by Genotype

\begin{tabular}{|c|c|c|c|c|c|c|}
\hline \multicolumn{7}{|c|}{ Male subjects } \\
\hline & & MAOA-I & & & MAOA-1 & \\
\hline \multirow[t]{2}{*}{$n$} & & 11 & & & 22 & \\
\hline & distrib. & $\begin{array}{l}\text { average } \\
\text { uncond. } \\
\text { contrib. }\end{array}$ & $\begin{array}{l}\text { average } \\
\text { belief }\end{array}$ & distrib. & $\begin{array}{l}\text { average } \\
\text { uncond. } \\
\text { contrib. }\end{array}$ & $\begin{array}{l}\text { average } \\
\text { belief }\end{array}$ \\
\hline Free-riders & $18.2 \%$ & 4.00 & 8.00 & $4.5 \%$ & 0 & 19.00 \\
\hline $\begin{array}{l}\text { Conditional } \\
\text { cooperators }\end{array}$ & $72.7 \%$ & 13.88 & 10.13 & $50.0 \%$ & 9.45 & 9.27 \\
\hline $\begin{array}{l}\text { Triangle } \\
\text { contributors }\end{array}$ & $0.0 \%$ & $\mathrm{n} / \mathrm{a}$ & $\mathrm{n} / \mathrm{a}$ & $22.7 \%$ & 7.80 & 7.40 \\
\hline Others & $9.1 \%$ & 10.00 & 10.0 & $22.7 \%$ & 9.20 & 10.20 \\
\hline
\end{tabular}

The difference in the proportion of conditional cooperators $(72.7 \%$ for MAOA- $L$ and $50 \%$ for $M A O A-H)$ was also insignificant ( $p=0.278$, Fisher's exact test). Within this behavioral type, we found that the average unconditional contribution of MAOA-L carriers (mean = 13.88) was higher than that of $M A O A-H$ genotypes (mean $=9.45)$, but again, the difference was not statistically significant ( $p=0.066, \mathrm{M}-\mathrm{W}$ test). The same was true for the average belief about others' contributions ( $p=0.867, \mathrm{M}-\mathrm{W}$ test). The share of triangle cooperators varied not significantly ( $p=0.143$, Fisher's exact test) across genetic predisposition, although no MAOA- $L$ carrier belonged to the type of triangle cooperators, whereas $22.7 \%$ of MAOA-H subjects did. The same was true for the difference between shares of others $(p=0.637$, Fisher's exact test).

To test whether the distribution of player types among female subjects differed between genotypes, we compared respective shares. We did not find a significant difference for freeriders $(p=1.00)$, conditional cooperators $(p=0.475)$, or triangle contributors $(p=0.580$, Fisher's exact test). MAOA-L female carriers belonged more often to the group others, which was characterized by very different behavioral patterns (such as a decreasing path or constant positive contributions), indicating a higher degree of heterogeneity among this genotype. The share, however, did not vary significantly across genetic predisposition ( $p=0.056$, Fisher's exact test). Furthermore, average unconditional contributions and beliefs did not differ significantly within player types and across genotypes. 
Table 2: Distribution of Female Player Types Separated by Genotype

\section{Female subjects}

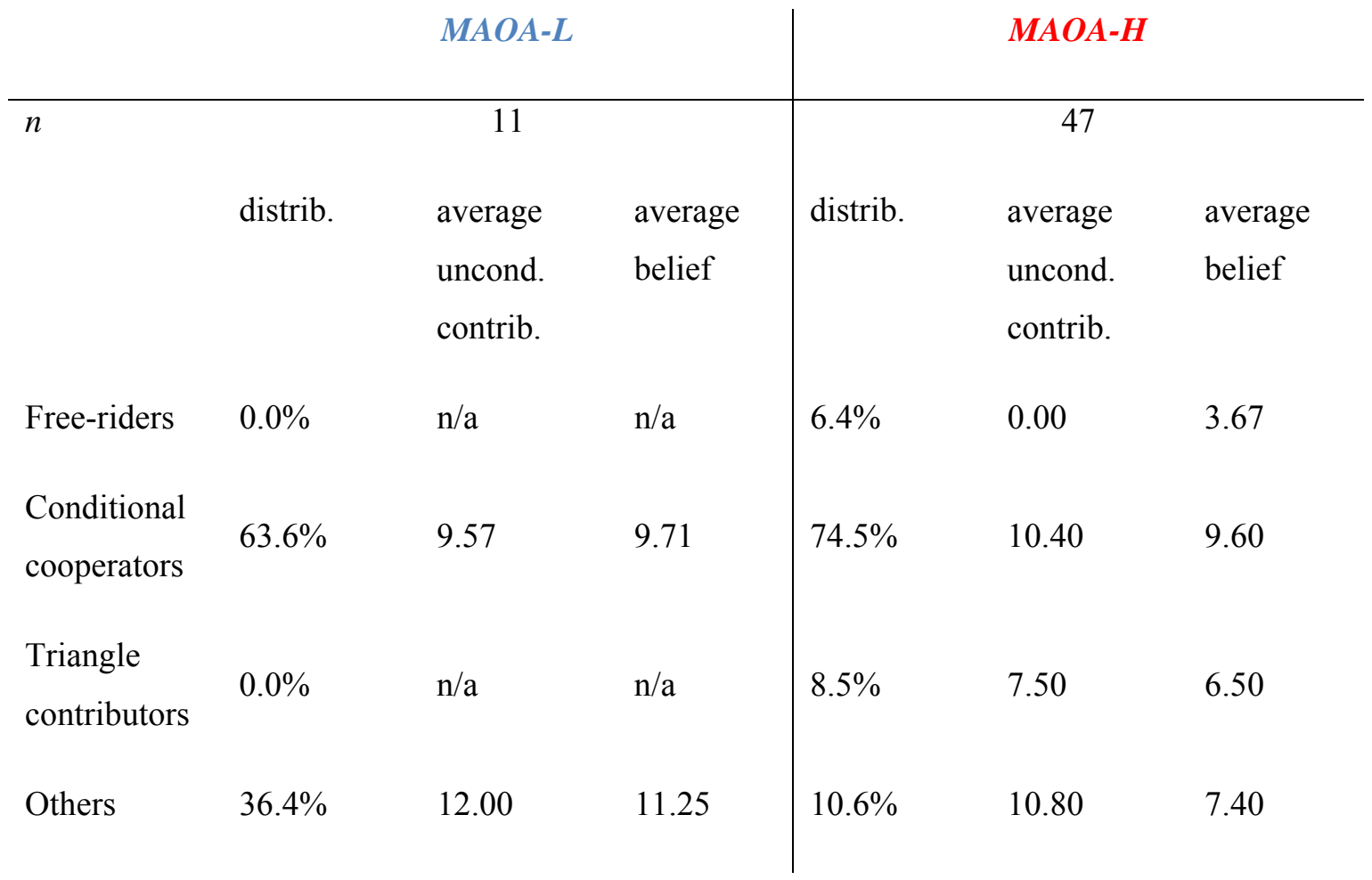

Previous studies, however, have suggested different classification schemes. In order to capture different player types' general tendencies, Kurzban and Houser (2001) suggested assigning to each subject the type that was consistent with their greatest number of moves. This procedure allows for some heterogeneity in each type's play and accommodates the fact that free-riders (in a wider sense) occasionally contribute some part of their endowment, while conditional cooperators occasionally contribute less than the "ideal" level. Whereas the classification of conditional cooperators proposed by Fischbacher et al. (2001) is able to capture some degree of heterogeneity due to the application of the Spearman rank correlation coefficient, this is not the case for free-riders. Therefore, we applied an alternative classification for this type. We used Kurzban and Houser's (2001) scheme to determine a contribution as being consistent with the free-rider type if it is less than $20 \%$ of the endowment (i.e., less than four) independent of the average group contribution. We then simply counted the number of decisions consistent with the free-rider rule. If the majority of all decisions were consistent with it, we assigned the type weak free-rider to the subject. 
Table 3: Shares of Weak Free Riders Separated by Genotype

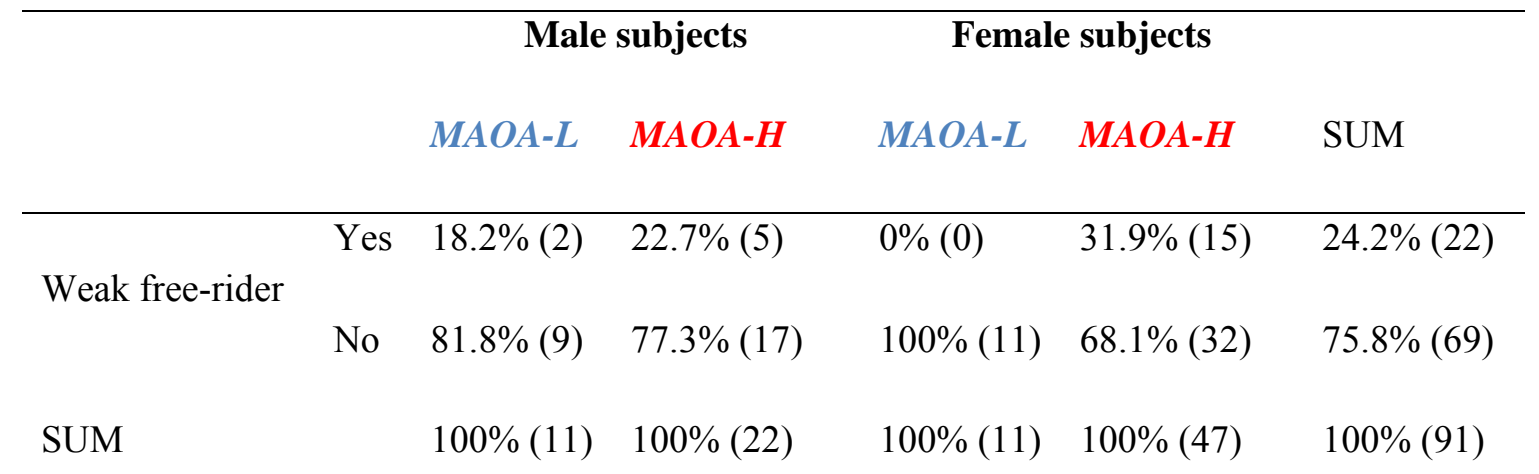

As can be seen in Table 3, this assignment resulted in about $24 \%$ belonging to the group of weak free-riders. For male subjects, the share of weak free-riders varied only slightly across genetic predisposition (18.2 vs. $22.7 \%$ ). Not surprisingly, the difference was insignificant ( $p$ $=1.00$, Fisher's exact test). For female subjects, however, a clear-cut picture emerged: whereas no MAOA-L carriers behaved like weak free-riders, $31.9 \%$ of MAOA-H subjects belonged to this group. The difference in the share across genetic predisposition is weakly significant ( $p=0.05$, Fisher's exact test). Therefore, we can state that the genotype has some predictive power in explaining the player type: female subjects carrying $M A O A-L$ are less likely to behave like weak free-riders than MAOA-H subjects.

As seen before, by applying the strict free-rider definition of zero contribution, we did not detect a significant difference between female genotypes. Results for the wider definition (very low contributions), however, were somewhat different and pointed to a link between genotype and behavioral type. This brings up the question of whether this finding is a technical artifact of this particular classification scheme. This is of particular importance, as there seems to be agreement that players use strategies that differ in systematical and discernible ways, but no consensus on the correct classification scheme (Kurzban \& Houser, 2001). Thus, all schemes are, to some extent, arbitrary. Therefore, as a robustness check, we varied how weak free-rider was categorized in that we considered the subjects' average contribution within the contribution table (mean of 21 decisions). We tested whether the share of weak free-riders varied across genetic predispositions when we applied a threshold of $20 \%$ (four tokens). We found that the difference was significant only at a $10 \%$ level $(p=0.097$, Fisher's exact test). A similar statistical difference occurred when we assumed a threshold of $15 \%$ or three tokens ( $p=0.097$, Fisher's exact test). Decreasing the threshold further yielded no difference, as already detected by applying a threshold of zero. Therefore, we can conclude that our data suggest a moderate association between weak free-riding and MAOA for female subjects. This link can be found by applying various player type classifications. However, 
there is neither a correlation between strong free-riding (i.e., zero or very little mean contributions) and MAOA for females, nor any link between MAOA and the occurrence of free-riding among males. Further research with larger samples is needed to establish a clearer picture of this potential link.

\subsection{Questionnaire Data}

We administered an extensive ex-post questionnaire for two different reasons. First, we intended to study our variable of interest, cooperation, in a different way to determine the robustness of our experimental results. Second, we intended to exclude the possibility that behavioral differences between genotypes are due to (exogenous) socio-economic background variables or (endogenous) personality variables. To verify the latter, we administered a questionnaire on background characteristics (e.g., age, field of study (economics yes/no) and number of friends) within an experimental session, as well as various personality measures. As all of these variables have previously been shown to affect individuals' level of cooperation, we tested whether the groups of MAOA-L and MAOA-H were comparable in terms of their background characteristics. We verified that behavioral differences between genotypes could not be attributed to differences in exogenous and endogenous background characteristics, as they did not differ at a $5 \%$-level between MAOA-H and MAOA-L for male and female subjects (see Table 4).

Table 4: Background Characteristics Across Genotypes

\begin{tabular}{|c|c|c|c|c|c|c|}
\hline & \multicolumn{3}{|c|}{ Male } & \multicolumn{3}{|c|}{ Female } \\
\hline & MAOA-L & MAOA-H & $p$-value & MAOA-L & MAOA-H & $p$-value \\
\hline Age & 25.45 & 24.82 & 0.381 & 24.91 & 24.53 & 0.825 \\
\hline Friends & 0.27 & 0.50 & 0.635 & 0.55 & 0.26 & 0.455 \\
\hline Economics (1=yes) & 0.27 & 0.41 & 0.703 & 0.18 & 0.28 & 0.710 \\
\hline Risk aversion ( 0 to 10 ) & 4.36 & 5.50 & 0.272 & 5.55 & 5.00 & 0.414 \\
\hline Patience ( 0 to 10$)$ & 5.82 & 5.18 & 0.460 & 4.45 & 4.81 & 0.734 \\
\hline Impulsivity ( 0 to 10$)$ & 4.91 & 3.91 & 0.272 & 5.00 & 4.72 & 0.747 \\
\hline Self-monitoring (0 to 18 ) & 10.73 & 9.14 & 0.191 & 8.64 & 8.06 & 0.506 \\
\hline Conscientiousness ( 1 to 7 ) & 4.45 & 4.83 & 0.767 & 5.21 & 5.18 & 0.894 \\
\hline Neuroticism (1 to 7) & 4.39 & 3.65 & 0.149 & 4.42 & 4.58 & 0.691 \\
\hline Openness ( 1 to 7 ) & 5.15 & 4.98 & 0.727 & 4.58 & 4.99 & 0.525 \\
\hline Agreeableness ( 1 to 7 ) & 5.18 & 5.08 & 0.873 & 4.79 & 4.98 & 0.506 \\
\hline Extroversion (1 to 7$)$ & 4.36 & 4.76 & 0.399 & 5.00 & 4.70 & 0.626 \\
\hline Positive reciprocity ( 1 to 7 ) & 6.15 & 6.11 & 0.583 & 6.33 & 6.16 & 0.393 \\
\hline Negative reciprocity (1 to 7 ) & 3.70 & 3.71 & 0.962 & 3.39 & 3.19 & 0.518 \\
\hline Machiavellianism (1 to 7) & 2.96 & 2.59 & 0.503 & 2.42 & 2.73 & 0.068 \\
\hline
\end{tabular}


With regard to the first aim (i.e., illuminating the link between genotype and player type in a non-experimental way), we measured individuals' willingness to cooperate by making use of a standardized, psychologically validated 20-item questionnaire on Machiavellianism (Christie \& Geis, 1970). The questionnaire data showed that female MAOA-L carriers, who have already been shown to be less likely to free-ride, had lower scores on the Machiavellianism scale than MAOA- $H$ individuals at a significance level of 6.8 $\%$ (M-W test, two-sided). Thus, we found that — consistent with our experimental findingsMAOA-L female carriers had lower scores on the Machiavellianism scale than MAOA-H individuals, indicating that the former are more likely to conform to a social norm of cooperation rather than to pursue their self-interest. As we found only Machiavellianism, but not other psychological and socio-demographic factors we measured, to be associated with $M A O A$, this finding reinforces the notion that the predictive power of genotype is not spurious. In addition, it provides further support for $M A O A$ as a genetic predictor of cooperative behavior.

For males, however, we did not observe such a relationship ( $p=0.503, \mathrm{M}-\mathrm{W}$ test, two-sided) which is in line with the findings from the strategic public goods experiment. To conclude, the association between the low activity allele of MAOA within females and Machiavellianism suggests an additional pathway between genes and cooperation.

\section{Discussion}

In 2000, Ben-Ner and Putterman realized that biologists' distinction between genotypes and phenotypes would be especially useful for the study of human preferences, but it took about a decade until the first efforts to make such a distinction were visible. At the same time, it is important to reconsider that it has long been routine to predict outcomes conditional on personal characteristics such as gender, health, and race that are themselves partially determined by genes (Manski 2011). Hence, Manski (2011) argued that one may now similarly predict outcomes conditional on gene measurements. With our study, we address an increasing demand for a deeper understanding of genetic - in addition to environmentalfactors that shape human behavior. In particular, we are not aware of any other attempt to link genotype and player type.

Recent findings in genoeconomics using both twin studies (e.g. Cesarini et al., 2009b) and genetic association designs (e.g., Knafo et al., 2008) have shown a genetic component to the other-regarding behavior revealed in economic games. From this point of view, searching for a genetic basis for the observed variation in individual preferences seems to be a 
promising avenue of research. We complement the literature on the (outside economics) widely-studied MAOA testing for an association of this candidate gene and behavior in a strategic public goods game. We offer initial evidence that the free-riding strategy is associated with MAOA gene variants in females. Our findings suggest a moderate effect of genetics on individuals' preferences towards cooperation: in females, genotype can, to some extent, predict player type. Female subjects carrying $M A O A-L$ are significantly less likely to be weak free-riders than $M A O A-H$ subjects. This finding is true for various classification schemes of the weak free-rider type. Furthermore, the association between genotypes of $M A O A$ in females and preferences towards cooperation seems to be independent of the elicitation procedure. Indeed, we not only found a link between a particular genetic variant and females' behavior in an incentivized public goods experiment, but also between this gene and responses in a non-incentivized personality questionnaire testing Machiavellianism. This reinforces our finding that $M A O A-L$ female carriers are more cooperative than MAOA-H carriers. Furthermore, $M A O A-L$ female subjects are significantly more cooperative than carriers of the highly efficient alleles in the presence of others who contribute little or nothing. Taken together, these results corroborate our previous findings (Mertins et al., 2011) using different elicitation procedures. In particular, by investigating behavior in a setting where there is no uncertainty, we ensure that behavioral differences do not stem from differences in expectations.

Recently, Chen and colleagues reported MAOA-L's predictive effort on happiness in female carriers and argued that MAOA- $L$ women may be more sensitive to positive aspects of their environment (Chen et al., 2012). This rather positive motivation of MAOA-L women together with higher levels of serotonin in the brain of MAOA-L carriers corroborate our finding of higher cooperativeness in MAOA-L females. However, more work needs to be done before we can tentatively report a true association.

With respect to male subjects, we found no statistically significant difference between both genotypes. We observed, however, a potentially interesting pattern in the data. If the other group members are willing to contribute large shares, MAOA-L and MAOA-H carriers develop in opposite directions: while $M A O A-L$ males still increase their contributions as others increase their average contributions, $M A O A-H$ subjects remain constant at a lower level. The difference is at its greatest when all group members contribute their full endowment. Further research is needed to clarify the non-significant results. Is there indeed no association for males, or do the null results stem from the relatively small number of subjects? A replication study using a somewhat larger sample size might render the results 
from just under the significance level to above. Although the observed tendency should not be over-interpreted, it points to a suggestive pattern in the data: MAOA-L male subjects are more cooperative than $M A O A-H$ subjects in a highly cooperative environment. Instead, we expected $M A O A-L$ carriers to be susceptible to free-riding rather than cooperation. An association to pro-social behavior is surprising at first glance, as it has been repeatedly been shown that $M A O A-L$ male carriers are susceptible to a variety of antisocial behaviors, including physical violence and criminal behavior. These findings recently motivated Beaver et al. (2010) to examine the association between MAOA and gang membership. They found that the low MAOA activity alleles conferred an increased risk of joining a gang for males, but not for females. Indeed, an important aspect of gang membership is the gang members' tendency for violence. Another aspect, however, is their feeling of solidarity and their willingness to be there for each other. In this regard, an exceptionally high level of cooperation in a group of highly pro-social others is not contradictory at all. Rather, our findings might contribute to a comprehensive picture that requires further exploration.

Our genoeconomic approach enlightens our understanding of individuals' heterogeneity. Identifying causes, genetic and/or environmental, is crucial because it may help to explain differences and similarities between individuals and/or groups. Fergusson and colleagues demonstrate that the combination of a range of adverse environmental and personal factors together with the MAOA- $L$ variant, but not genotype alone, triggers antisocial behavior (Fergusson et al., 2012). Brain imaging studies clearly show that functional genetic variants of $M A O A$ a) have an effect on the brain development; b) are modulators of environmental factors in terms of gene-environment interactions, which often display sex differences; and c) result in developmental alterations to specific neuronal circuits, causing different responses to external stimuli (Nordquist \& Oreland, 2010). However, the fact that one single variant in a given gene is (moderately) associated with variability in a given trait should not be over-interpreted. Genoeconomics is a nascent field and a frontier area. Many more studies are needed to understand the neural underpinnings of pro-social behavior. Genoeconomics, together with neuroscience, may help to identify causes, either genetic, environmental, or both (Navarro, 2009). We are convinced that a better understanding of all determinants of cooperative behavior and their interactions will benefit economic modeling and will inform policy analysis. In this regard, our study is the first step towards establishing a link between genes and cooperation preferences. Further research is needed to clarify the exact role of MAOA and other genes in transmitting social preferences and their interactions with each other and with the environment in males and females. 


\section{References}

Alford, J., Funk, C., \& Hibbing, J. R. (2005). Are political orientations genetically transmitted? American Political Science Review, 99(2), 153-167.

Andersen, S., Bulte, E., Gneezy, U., \& List, J. A. (2008). Do women supply more public goods than men? Preliminary experimental evidence from matrilineal and patriarchal societies. American Economic Review, 98(2), 376-381.

Anderson, L. R., DiTraglia, F. J., \& Gerlach, J. R. (2011). Measuring altruism in a public goods experiment: A comparison of U.S. and Czech subjects. Experimental Economics, 14(3), 426437.

Apicella, C. L., Dreber, A., Gray, P. B., Hoffman, M., Little, A. C., \& Campbell, B. C. (2011). Androgens and competitiveness in men. Journal of Neuroscience, Psychology, and Economics, 4, 54-62.

Apicella C. L., Cesarini D., Johannesson M., Dawes C. T., Lichtenstein P., Wallace, B., et al. (2010). No association between oxytocin receptor (OXTR) gene polymorphisms and experimentally elicited social preferences. PLOS ONE, 5: e11153.

Beauchamp, J. P., Cesarini, D., Johannesson, M., van der Loos, M.J.H.M., Koellinger, P. D., et al. (2011). Molecular Genetics and Economics. Journal of Economic Perspectives, 25(4), 57-82.

Beaver, K. M., DeLisi, M., Vaughn, M. G., \& Barnes, J. C. (2010). Monoamine Oxidase A genotype is associated with gang membership and weapon use. Comprehensive Psychiatry, 51(2), 130 134.

Behrman, J. R., \& Taubman, P. (1989). Is schooling 'mostly in the genes'? Nature-nurture decomposition using data on relatives. Journal of Political Economy, 97(6), 1425-1446.

Benjamin, D. J., Chabris, C. F., Glaeser, E. L., Gudnason, V., Harris, T. B., et al. (2007). Genoeconomics. In M. Weinstein, J. W. Vaupel, \& K. W. Wachter (Eds.), Biosocial Surveys (pp. 192-289). Washington: National Academies Press.

Benjamin, D. J., Cesarini, D., van der Loos, M. J. H. M., Dawes, C. T., Koellinger, P. D., Magnusson, P. K. E., Chabris, C.F., Conley, D., Laibson, D., Johannesson, M., \& Visscher, P.M. (2012): The genetic architecture of economic and political preferences. Proceedings of the National Academy of Sciences, 109(21), 8026-8031.

Ben-Ner, A., \& Putterman, L. (2000). On some implications of evolutionary psychology for the study of preferences and institutions. Journal of Economic Behavior and Organization, 43, 91-99.

Brandts, J., \& Charness, G. (2000). Hot vs. cold: Sequential responses and preference stability in experimental games. Experimental Economics, 2, 227-238.

Brosig, J. (2002). Identifying cooperative behavior: Some experimental results in a prisoner's dilemma game. Journal of Economic Behavior and Organization, 47, 275-290.

Brosig, J., Weimann, J., \& Yang, C. (2003). The hot versus cold effect in a simple bargaining experiment. Experimental Economics, 6, 75-90.

Burlando, R., \& Guala, F. (2005). Heterogeneous agents in public goods experiments. Experimental Economics, 8(1), 35-54.

Burlando, R., \& Hey, J. D. (1997). Do Anglo-Saxons free-ride more? Journal of Public Economics, 64(1), 41-60.

Buser, T. (in press). The impact of the menstrual cycle and hormonal contraceptives on competitiveness. Journal of Economic Behavior and Organization.

Cases, O., Seif, I., Grimsby, J., Gaspar, P., Chen, K., et al. (1995). Aggressive behavior and altered amounts of brain serotonin and norepinephrine in mice lacking MAOA. Science, 268(5218), $1763-1766$ 
Caspi, A., McClay, J., Moffitt, T. E., Mill, J., Martin, J., et al. (2002). Role of genotype in the cycle of violence in maltreated children. Science, 297, 851-854.

Cesarini, D., Dawes, C. T., Fowler, J. H., Johannesson, M., Lichtenstein, P., \& Wallace, B. (2008). Heritability of cooperative behavior in the trust game. Proceedings of the National Academy of Sciences of the United States of America, 105(10), 3721-3726.

Cesarini, D., Dawes, C. T., Johannesson, M., Lichtenstein, P., \& Wallace, B. (2009a). Genetic variation in preferences for giving and risk taking. The Quarterly Journal of Economics, 124(2), 809-842.

Cesarini, D., Dawes, C. T., Johannesson, M., Lichtenstein, P., \& Wallace, B. (2009b). Experimental game theory and behavior genetics. Annals of the New York Academy of Sciences, 1167, 6675 .

Chaudhuri, A. (2011). Sustaining cooperation in laboratory public goods experiments: A selective survey of the literature. Experimental Economics, 14, 47-83.

Chen, H., Pine, D. S., Ernst, M., Gorodetsky, E., Kasen, S., Gordon, K., Goldman, D., \& Cohen, P. (2012). The MAOA gene predicts happiness in women. Progress in NeuroPsychopharmacology \& Biological Psychiatry, 40, 122-125. doi: 10.1016/j.pnpbp.2012.07.018.

Christie, R., \& Geis, F. L. (1970). Studies in Machiavellianism. New York: Academic Press.

Costa, P. T., \& McCrae, R. R. (1989). The NEO PI/FFI manual supplement. Odessa (Florida): Psychological Assessment Resources.

Craig, I. W. (2007). The importance of stress and genetic variation in human aggression. Bioessays, 29(3), 227-236.

Croson, R., \& Gneezy, U. (2009). Gender differences in preferences. Journal of Economic Literature, $47(2), 448-474$.

Decety, J., Jackson, P. L., Sommerville, J. A., Chaminade, T., \& Meltzoff, A. N. (2004). The neural bases of cooperation and competition: An fMRI investigation. NeuroImage, 23, 744- 751.

De Neve, J.-E., Christakis, N., Fowler, J., \& Frey, B.S. (2012). Genes, economics, and happiness. Journal of Neuroscience, Psychology, and Economics, 5(4), 193-211.

Dohmen, T., Falk, A., Huffman, D., \& Sunde, U. (2009). Homo reciprocans: Survey evidence on behavioral outcomes. Economic Journal, 119, 592-612.

Dreber A., Apicella, C. L., Eisenberg, D. T. A., Garcia, J. R., Zamore, R., et al. (2009). The 7R polymorphism in the dopamine receptor D4 gene (DRD4) is associated with financial risk taking in men. Evolution and Human Behavior, 30(2), 85-92.

Dreber A., Rand D., Wernerfelt N., Garcia J., Vilar M., et al. (2011). Dopamine and risk choices in different domains: Findings among serious tournament bridge players. Journal of Risk and Uncertainty, 43, 19-38.

Fehr, E., Bernhard, H., \& Rockenbach, B. (2008). Egalitarianism in young children. Nature, 454, 1079-1083.

Fehr, E., \& Fischbacher, U. (2004). Social norms and human cooperation. Trends in Cognitive Sciences, 8(4), 185-190.

Fergusson D. M., Boden, J. M., Horwood, L. J., Miller, A., \& Kennedy, M. A. (2012). Moderating role of the MAOA genotype in antisocial behaviour. British Journal of Psychiatry, 200(2), 116-23. doi: 10.1192/bjp.bp.111.093328.

Fischbacher, U. (2007). Z-tree: Zurich toolbox for ready-made economic experiments. Experimental Economics, 10(2), 171-178.

Fischbacher, U., \& Gächter, S. (2010). Social preferences, beliefs, and the dynamics of free riding in public goods experiments. American Economic Review, 100(1), 541-556. 
Fischbacher, U., Gächter, S., \& Fehr, E. (2001). Are people conditionally cooperative? Economics Letters, 71(3), 397-404.

Frydman, C., Camerer, C., Bossaerts, P., \& Rangel, A. (2011). MAOA-L carriers are better at making optimal financial decisions under risk. Proceedings of the Royal Society, 278(1714), 20532059.

Gallardo-Pujol, D., Andrés-Pueyom A., \& Maydeu-Olivares, A. (forthcoming). MAOA genotype, social exclusion and aggression: An experimental test of a gene-environment interaction. Genes Brain Behavior.

Gerlitz, J.Y., \& Schupp, J. (2005). Zur Erhebung der Big-Five-basierten Persönlichkeitsmerkmale im SOEP. DIW Research Notes, 4.

Graf, A. (2004). Eine deutschsprachige Version der Self-Monitoring-Skala. Zeitschrift für Arbeits- und Organisationspsychologie, 48(3), 109-121.

Greiner, B., 2004. An online recruitment system for economic experiments. In K. Kremer, \& V. Macho (Eds.), Forschung und wissenschaftliches Rechnen 2003 (pp. 79-93), GWDG Bericht, Vol. 63, Göttingen: Gesellschaft für Wissenschaftliche Datenverarbeitung.

Güth, W., Huck, S., \& Müller, W. (2001). The relevance of equal splits in ultimatum games. Games and Economic Behavior, 37, 161-169.

Gunnthorsdottir, A., McCabe, K., \& Smith, V. (2002). Using the Machiavellianism instrument to predict trustworthiness in a bargaining game. Journal of Economic Psychology, 23, 49-66.

Hariri, A. R., Drabant, E. M., Munoz, K. E., Kolachana, B. S., Mattay, V. S., Egan, M. F., \& Weinberger, D. R. (2005). A susceptibility gene for affective disorders and the response of the human amygdala. Archives of General Psychiatry, 62(2), 146-152.

Herrmann, B., \& Thöni, C. (2009). Measuring conditional cooperation: A replication study in Russia. Experimental Economics, 12(1), 87-92.

Hines, M. (2011). Gender Development and the Human Brain. Annual Reviews of Neuroscience, 34(1), 69-88.

Israel, S., Lerer, E., Shalev, I., Uzefovsky, F., Riebold, M., et al. (2009). The oxytocin (OXTR) contributes to prosocial fund allocations in the dictator game and the social value orientations task. PLoS One, 4(5): e5535.

Kim-Cohen, J., Caspi, A., Taylor, A., Williams, B., Newcombe, R., Craig, I. W., \& Moffitt, T. E. (2006). MAOA, maltreatment, and gene-environment interaction predicting children's mental health: new evidence and a meta-analysis. Molecular Psychiatry 11(10), 903-913.

Knafo, A., Israel, S., Darvasi, A., Bachner-Melman, R., Uzefovsky, F., et al. (2008). Individual differences in allocation of funds in the dictator game associated with length of the arginine vasopressin 1a receptor (AVPR1a) RS3 promoter-region and correlation between RS3 length and hippocampal mRNA. Genes, Brain and Behavior, 7(3), 266-275.

Kocher, M. G., Cherry, T., Kroll, S., Netzer, R. J., \& Sutter, M. (2008). Conditional cooperation on three continents. Economics Letters, 101(3), 175-178.

Kuhnen, C. M., \& Chiao, J. Y. (2009). Genetic determinants of financial risk taking. PLoS ONE, 4(2): e4362. doi:10.1371/journal.pone.0004362.

Kuo, J., Hamid, N., Bondar, G., Prossnitz, E.R., \& Micevych, P. (2010). Membrane estrogen receptors stimulate intracellular calcium release and progesterone synthesis in hypothalamic astrocytes. The Journal of Neuroscience, 30(39), 12950-12957

Kurzban, R., \& Houser, D. (2001). Individual differences in cooperation in a circular public goods game. European Journal of Personality, 15(S1), S37-S52. 
Kurzban, R., \& Houser, D. (2005). Experiments investigating cooperative types in humans: A complement to evolutionary theory and simulations. Proceedings of the National Academy of Sciences of the United States of America, 102(5), 1803-1807.

Liu, D., Diorio, J., Tannenbaum, B., Caldji, C., Francis, D., Freedman, A., Sharma, S., Pearson, D., Plotsky, P. M., \& Meaney, M. J. (1997). Maternal care, hippocampal glucocorticoid receptors, and hypothalamic-pituitary-adrenal responses to stress. Science, 277(5332), 1659-1662.

Manski, C. (2011). Genes, eyeglasses, and social policy. Journal of Economic Perspectives, 25(4), 8394.

McDermott, R., Tingley, D., Cowden, J., Frazzetto, G., \& Johnson, D. (2009). Monoamine oxidase a gene $(M A O A)$ predicts behavioral aggression following provocation. Proceedings of the National Academy of Sciences of the United States of America, 106(7), 2118-2123.

Meaney, M. J. (2001). Maternal care, gene expression, and the transmission of individual differences in stress reactivity across generations. Annual Review of Neuroscience, 24, 1161-1192.

Mertins, V., Schote, A., Hoffeld, W., Griessmair, M., \& Meyer, J. (2011). Genetic susceptibility for individual cooperation preferences: The role of monoamine oxidase A gene (MAOA) in the voluntary provision of public goods. PLOS ONE, 6(6): e20959.

Meyer-Lindenberg, A., Buckholtz, J.W., Kolachana, B., Hariri, A.R., Pezawas, L., Blasi, G., Wabnitz, A., Honea, R., Verchinski, B., Callicott, J.H., Egan, M., Mattay, V., \& Weinberger, D.R.(2006). Neural mechanisms of genetic risk for impulsivity and violence in humans. Proceedings of the National Academy of Sciences USA, 103, 6269-6274

Navarro, A. (2009). Genoeconomics: Promises and caveats for a new field. Annals of the New York Academy of Sciences, 1167, 57-65.

Nelson, R. J., \& Trainor, B. C. (2007). Neural mechanisms of aggression. Nature Reviews Neuroscience, 8(7), 536-546,

Nicolaou, N., \& Shane, S. (2010). Entrepreneurship and occupational choice: Genetic and environmental influences. Journal of Economic Behavior and Organization, 76, 3-14.

Nordquist, N., Oreland, L. (2010). Serotonin, genetic variability, behaviour, and psychiatric disorders a review. Upsala Journal of Medical Sciences 115 (1), 2-10.

Ostrom, E. (2000). Collective action and the evolution of social norms. Journal of Economic Perspectives, 14(3), 137-158.

Ou, X. M., Chen, K., \& Shih, J. C. (2006). Glucocorticoid and androgen activation of monoamine oxidase A is regulated differently by R1 and Sp1. Journal of Biological Chemistry, 281, 21512-21525.

Reif, A., Scarpini, E., Venturelli, E., Töpner, T., Fenoglio, C., Lesch, K.-P, \& Galimberti, D. (2008). The functional MAOA-uVNTR promoter polymorphism in patients with frontotemporal dementia. European Journal of Neurology, 15(6), 637-639.

Riecher-Rossler, A., \& Geyter, C. de (2007). The forthcoming role of treatment with oestrogens in mental health. Swiss Medical Weekly, 137(41-42), 565-572.

Rommelse, N.N., Altink, M.E., Arias-Vasquez, A., Buschgens, C.J., Fliers, E., Faraone SV. Buitelaar,J.K., Sergeant,J.A., Oosterlaan J., \& Franke, B. (2008) Differential association between MAOA, ADHD and neuropsychological functioning in boys and girls. American Journal of Medical Genetics Part B: Neuropsychiatric Genetics, 147B, 1524-1530.Roth, A. (1995). Bargaining experiments. In J. Kagel \& A. Roth (Eds.), The Handbook of Experimental Economics (pp. 253-348). Princeton: Princeton University Press.

Sabol, S. Z., Hu, S., \& Hamer, D. (1998). A functional polymorphism in the monoamine oxidase A gene promoter. Human Genetics, 103(3),273-239. 
Selten, R. (1967). Die Strategiemethode zur Erforschung des eingeschränkt rationalen Verhaltens im Rahmen eines Oligopolexperiments. In H. Sauermann (Ed.), Beiträge zur experimentellen Wirtschaftsforschung (pp. 136-168). Tübingen: J.C.B. Mohr Paul Siebeck.

Shajek, A. (2007). Entwicklung zweier Kurzskalen zur Messung von Machiavellismus und Egoismus. Dokumentation eines Instrumententests auf Basis des SOEP-Pretests 2006. DIW Research Notes, 22.

Sjöberg, R. L., Nilsson, K. W., Wargelius, H. L., Leppert, J., Lindström, L., \& Oreland, L. (2007). Adolescent girls and criminal activity: Role of MAOA-LPR genotype and psychosocial factors. American Journal of Medical Genetics Part B: Neuropsychiatric Genetics, 144B(2), 159-164.

Snyder, M. (1974). Self-monitoring of expressive behavior. Journal of Personality and Social Psychology, 30, 526-537.

Soares, M.C., Bshary, R., Fusani, F., Goymann, W., Hau, M., Hirschenhauser, K., \& Oliveira R.F. (2010). Hormonal mechanisms of cooperative behavior. Philosophical Transactions of the Royal Society B: Biological Science, 365(1553), 2737-2750.

Spitzer, M., Fischbacher, U., Herrnberger, U., Grön, G., \& Fehr, E. (2007). The neural signature of social norm compliance. Neuron, 56(1), 185-196.

Taubman, P. (1976). The determinants of earnings: Genetics, family, and other environments: A study of white male twins. American Economic Review, 66(5), 858-870.

Wallace, B., Cesarini, D., Lichtenstein, P., \& Johannesson, M. (2007). Heritability of ultimatum game responder behavior. Proceedings of the National Academy of Sciences of the United States of America, 104(40), 15631-15634.

Wright, N. D., Bahrami, B., Johnson, E., Di Malta, G., Rees, G., Frith, C. D.,\& Dolan, R. J. (2011). Testosterone disrupts human collaboration by increasing egocentric choices. Proceedings of the Royal Society B: Biological Sciences, 279(1736), 2275-2280.

Zhong, S., Israel, S., Xue, H., Ebstein, R. P., \& Chew, S. H. (2009 a). Monoamine Oxidase A gene (MAOA) associated with attitude towards longshot risks. PLoS One, 4(12): e8516.

Zhong, S., Chew, S. H., Set, E., Zhang, J., Xue, H., et al. (2009 b). The heritability of attitude toward economic risk. Twin Research and Human Genetics, 12, 103-107.

Ziermans, T., Dumontheil, I.; Roggeman, C., Peyrard-Janvid, M., Matsson, H., Kere, J., \& Klingberg, T. (2012). Working memory brain activity and capacity link MAOA polymorphism to aggressive behavior during development. Translational Psychiatry, 2(2). e85. 


\section{Appendix}

\section{Genotyping}

DNA was obtained from buccal cells followed by a mouthwash with Listerine (Qiagen Gentra Puregene Buccal Cell Kit, Hilden, Germany). PCR was performed in $50 \mu 1$ reactions with a total DNA concentration of $100 \mathrm{ng}, 1.5 \mathrm{mM} \mathrm{MgCl}$, 10 pmol of each primer, $0.2 \mathrm{mM}$ dNTPs and 1.25 U Hot Star Taq Polymerase (Qiagen). MAOA primer sequences were previously described (Sabol et al. 1998): MAOA Fwd (5'-ACAGCCTGACCGTGGA-GAAG-3') and MAOA Rev (5'GAACGGACGCTCCATTCGGA- 3'). Thermal cycling was carried out using the following conditions: pre-step $15 \mathrm{~min}$ at $95^{\circ} \mathrm{C}, 5$ min denaturing step at $94^{\circ} \mathrm{C}, 40$ cycles at $94^{\circ} \mathrm{C}$ for $30 \mathrm{sec}, 63^{\circ} \mathrm{C}$ for $40 \mathrm{sec}, 72^{\circ} \mathrm{C}$ for $30 \mathrm{sec}$ and a final extension phase at $72^{\circ} \mathrm{C}$ for 7 min. PCR products were separated on a $2 \%$ agarose gel and visualised with ethidium bromide under UV light. The PCR products resulted in fragments of 291 bp, 321 bp, 336 bp, 351 bp and 381 bp corresponding to the 2-, 3-, 3.5-, 4- and 5-repeat alleles, respectively. Group definition for MAOA-L (low activity) and MAOA-H (high activity) were previously described (Reif et al. 2008). 


\section{IAAEU Discussion Paper Series in Economics}

01/2012 Relative Consumption Concerns or Non-Monotonic Preferences?

Inga Hillesheim and Mario Mechtel

02/2012 Profit Sharing and Relative Consumption

Laszlo Goerke

03/2012 Conspicuous Consumption and Communism: Evidence From East and West Germany Tim Friehe and Mario Mechtel

04/2012 Unemployment Benefits as Redistribution Scheme for Trade Gains - A Positive Analysis Marco de Pinto

05/2012 Failure of Ad Valorem and Specific Tax: Equivalence under Uncertainty Laszlo Goerke, Frederik Herzberg and Thorsten Upmann

06/2012 The Redistribution of Trade Gains and the Equity-Efficiency Trade-Off Marco de Pinto

07/2012 Trade Union Membership and Sickness Absence: Evidence from a Sick Pay Reform Laszlo Goerke and Markus Pannenberg

08/2012 Risk-Sorting and Preference for Team Piece Rates

Agnes Bäker and Vanessa Mertins

09/2012 Union Wage Setting and International Trade

Hartmut Egger and Daniel Etzel

10/2012 How Much Do Others Matter? Explaining Positional Concerns for Different Goods and Personal Characteristics

Inga Hillesheim and Mario Mechtel

11/2012 Benefit Morale and Cross-Country Diversity in Sick Pay Entitlements Daniel Arnold

01/2013 Relative Consumption and Tax Evasion

Laszlo Goerke

02/2013 Variants of the Monoamine Oxidase A Gene (MAOA) Predict Free-riding Behavior in Women in a Strategic Public Goods Experiment Vanessa Mertins, Andrea B. Schote and Jobst Meyer 